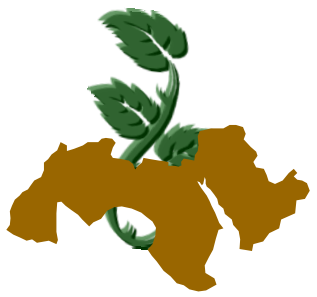

Arab Univ.

\title{
APPLICATION OF MACHINE VISION FOR DETECTION OF FOREIGN MATTER IN WHEAT GRAINS
}

\author{
Elbatawi ${ }^{1}$, I.E. and G.K. Arafa ${ }^{1}$ \\ 1- Agricultural Engineering Research Institute, P.O. Box 256, Nadi Elsaid St., Dokki, Giza, \\ Egypt
}

Keywords: Machine vision, Neural network, Foreign matter, Detection, Wheat

\section{ABSTRACT}

With the level of automation at every level of food production and the rates at which food is being produced, it is becoming increasingly more important to have systems that can automatically detect foreign matter along the way. The color scheme could make a computer vision system very practical for foreign object detection and removal. Research at laboratory levels has demonstrated that machine vision is an effective method for classification of cereal grains like wheat. Robust machine vision algorithms have been developed and tested to extract morphological, color and textural features of wheat grains and dockage content. The samples used in this study were bulk images of Egyptian wheat (Sakha8) mixed with known quantities of barley, rice and stones $(0.5 \%, 1.0 \%, 2.0 \%$ and $5 \%$ ). Back propagation neural network (BPNN) and statistical classifiers were used for classification. Results of the study indicate that classification was reduced from about $97 \%$ for wheat mixed with stones to $96 \%$ for wheat mixed with rice and $93 \%$ for wheat mixed with barley (at $5.0 \%$ admixture). This trend indicates that the features of $1.0 \%$ foreign matter admixture started overlapping with other classes (of admixture). On the other hand, $94 \%, 95 \%$ and $97 \%$ of $5 \%$ barley, rice and stones admixtures (with wheat) were accurately classified using neural network classifier. Using machine vision system, the detection rate for foreign matter in otherwise clean wheat was $100 \%$ with no false positives. This detection scheme was based on a linear feature detector incorporating two orthogonal masks.

\section{INTRODUCTION}

Egyptian Grain Commission has stringent regulations on the cleanliness and uniformity of wheat grain for both domestic and export grades. While cleaning, dockage is removed as it reduces the marketability and sale price of grains. Contaminants that remain in grain after the removal of dockage are called foreign matter. Egypt imposes a maximum tolerance limit of $2.5 \%$ of foreign matter (by weight) in export quality wheat (Ministry of Agriculture, 2003). Currently, foreign matter in a wheat sample is determined visually by grain inspectors. This is a tedious job and machine vision can play a major role in assisting grain inspectors in the determination of foreign material. For the near future, it is estimated that large amounts of food and agricultural products will be distributed throughout the world, thus the main food and agricultural products will be keenly competing in a free trade environment except for special or local products. In order to win the competition, it is necessary for food and agricultural products to have high quality and stringent quality control. It is especially important for food and agricultural products to have a high degree of safety. Safety inspections for quality control consist of biological, chemical and physical tests. The biological inspection is to detect contamination by microorganisms. The chemical inspection is to detect harmful substances like agricultural chemical residues. The physical inspection is to detect physical foreign materials embedded in the products (Muramatsu, 1989). However, as the type of foreign materials and their locations in the food product are usually unknown, there are no fixed physical inspection methods except for the detection of metallic foreign materials (Kenmochi, 1992). It is especially difficult to detect foreign ma- 
terials mixed in processed foods with a rapid nondestructive method as the amount and/or location of foreign materials are unknown. The best defense against foreign objects is to insure they do not get into the product in the first place. Common components of the strategy involve careful control of raw materials, minimizing inline components of the system that can break off and fall into the product and rigorously controlling objects that workers can have in the vicinity of a production line. One purpose of the study was to determine the characteristics of objects that may cause traumatic injury including laceration and perforation of tissues of the mouth, tongue, throat, stomach and intestine as well as damage to teeth and gums. These smaller objects are a risk to infants, surgery patients and the elderly. Unfortunately, about $80 \%$ of foreign object ingestion cases occur in children less than 3 years old (Stier, 2003). A significant amount of research has focused on the detection of metal, and systems can be purchased that very accurately detect and then remove any metal items that pass underneath. One solution in practice is to insert metal fibers into the plastic formulation so metal detectors will see pieces of belt. Another strategy is a policy that mandates a color for all objects in the production area that stands out from the product. Most of dry food like wheat grains is available in yellow color and very few food are blue, green, red or black colors. Bag liners, some foreign seeds, mud and conveyor parts are available in black colors that will stand out in bins of product. The hope is that line workers will recognize these bright colored objects in the product stream and remove them before they are shipped out. Monitoring is a vital part of any program control. However, scanning grain for particulate contaminants presents some problems. For grain in transit, only a few minutes can be available at intake within which to inspect a truck load. Also, contaminant particles will generally not be distributed uniformly through the load and grains (like wheat, barley, oats) are relatively low-value commodity compared to rice or nuts which are sold unprocessed to the consumer. Hence any method of scanning for contaminants in grain must be relatively inexpensive. Current methods are limited to human visual inspection of a grain sample, with sieving to aid the detection of foreign matter. When investigating the internal condition of various materials, X-rays are most effective. Although there are some studies in X-rays used for non-destructive inspection, there are few fundamental studies and hardly any for practical use (Morita et al 1996a). In general, the detection of foreign materials using $X$ rays is based on their absorption characteristics which vary with different material densities. It is possible to use Computer Tomography (CT) numbers from an X-ray CT system to measure different densities and components to detect foreign materials in food (Morita et al 1996b). However, CT numbers contain some systemic errors which occur during the measuring and computing phases of the image reconstruction process. Thus this system error (point spread function) can be a major problem in the practical detection of foreign materials in food. There are several challenges to fielding a vision system for detecting foreign objects in wheat grains. A computer vision system is much more effective at this task than human observers (Elbatawi, 2008). First, the vision system continuously watches the product stream and does not take breaks or blink like a human observer. Second, the vision system can freeze motion on a relatively high-speed belt and its resolution can be specifically tailored for the observation task at hand. These system capabilities allow the system to operate on a high-speed line that can separate product and present more surface area for screening. Finally, by automating the detection process, there is a digital record and alarm of the event that can help enforce policies to find and repair the cause of the foreign object in the first place. Optical sorting systems that use color or intensity as a basis for finding defective product and foreign matter have been in service for over 50 years for products like grains and teas (Daley and Carey, 1991). Color sorting systems capable of measuring shape, volume and other parameters are operating in fruit and vegetable processing (Elbatawi and Ebaid, 2006). Color vision has also been used for meat and poultry inspection to identify meat quality and identify systemic defects in carcasses (Petracci et al 2004). The identification and quantification of foreign matter in pure grain bulk with the developed algorithms is necessary as it will help to decide grain management. For this study, barley, rice and stones were added as a foreign matter in wheat. The objectives of this study are:

1 - To assess the ability of a machine vision system in classifying foreign matter (barley rice and stones) buried in the bag stacks of wheat grains. 2- To quantify the level of foreign matter from digital images using statistical and artificial neural network classifiers.

The strategy of this research was to test a prototype in the lab (this study), and when suitable performance was reached, the prototype can be 
developed onto a production line to test long-term performance (near future).

\section{MATERIALS AND METHODS}

\section{Samples}

Wheat like Sakha8, Sakha69, Giza168, and Sohag3 varieties are the most popular all over Egypt. Sakha8 variety conditioned to $12 \%$ storage moisture content (wet basis) was used as samples in this study (most available variety). One thousand kernels (about $200 \mathrm{~g}$ each) of wheat with 0.5, 1.0, 2.0 and $5 \%$ barley admixture were prepared in separate bags as shown in Fig. (1).

Each sample was mixed well and poured into a $15.0 \mathrm{~cm} \times 20.0 \mathrm{~cm}$ rectangular dish with $3.0 \mathrm{~cm}$ deep. Excess grains piled above the rim of the dish were gently removed by scraping with a steel ruler in two orthogonal directions. The same way (with same amount of samples) was used to prepare the mixture of wheat with rice and wheat with stones. All 1000 samples were imaged in the similar fashion.

\section{Vision system and processing}

The image acquisition system consisted of a three-chip charge coupled device (CCD) color camera (DXC-350A, Sony, Japan) with a zoom lens of 10-120 mm focal length, a personal computer (PC Pentium 3), color frame-grabbing board (Meteor-II PCl frame grabber, Matrix Electronic Systems Ltd, Osaka, Japan), and a diffuse illumination chamber. Illumination was provided by a fluorescent light source of $300 \mathrm{~mm}$ diameter, $35 \mathrm{~W}$ circular lamp (FC12T9/CW, Philips, Singapore) with a rated voltage of $220 \mathrm{~V}$ as shown in Fig. (2). A monitor (NTSC) composite color signal from the camera was converted by the camera control unit at a speed of 30 frames per second into three parallel analog red, green, and blue (RGB) video signals and a synchronous signal. The frame grabber installed in the PC digitized the RGB analog video signals from the camera control unit into three 8-bit 256 by 256 digital images as tagged image file format images. Machine vision algorithm developed and used by Paliwal et al (2003) was used to extract 200 (125 color and 75 textural) features from the 500 bulk images.

\section{Classification}

The image features were grouped into 5 sets at each barley rice and stones mixture level for classification purposes. The extracted 200 image features were reduced to 75 features using
STEPDISC function (SAS, 2000). The averagesquared-canonical correlation were used as the criteria of significance. The linear discriminant and non-parametric classifiers were developed using the DISCRIM procedure (SAS 2000). The discriminant analysis was used to determine the classification accuracy for all the 5 sets. Classification accuracies were determined by randomly selecting the training and testing sets. Five training and test data sets, each containing 300 data points for training and 100 for testing, were used for classification purpose. The average of the five trials was calculated as the mean classification accuracy. The classification accuracies were also determined using a four layer back-propagation neural network (BPNN). A neural network software package (Neuroshell 2, version 4.0, Ward Systems Group, Frederick, MD) was used for this purpose. Back propagation neural network (BPNN) classifier was designed for two different test scenarios i.e. full (75) features model, and top 25 features model. A worksheet file with input and output variables was prepared with first 100 patterns representing wheat with $0.5 \%$ barley (same way with rice and stones) marked as class 1 and so on up to class 4 representing wheat with $5 \%$ barley (same way with rice and stones). Average classification of five trials was calculated to draw bar graphs discussed in the following sections.

\section{RESULTS AND DISCUSSION}

\section{Classification using statistical classifiers}

Fig. (3) shows the mean classification accuracy at four different levels of barley, rice and stones mixed with wheat. Classification accuracy was reduced from $95 \%$ for wheat mixed with stones to $92 \%$ for wheat mixed with rice and $89 \%$ for wheat mixed with barley (at $0.5 \%$ admixture). On the other hand, classification accuracy becomes better at $5.0 \%$ admixture for all foreign matter mixed with wheat. For example, classification accuracy reduced from $97 \%$ for wheat mixed with stones to $96 \%$ for wheat mixed with rice and $93 \%$ for wheat mixed with barley (at $5.0 \%$ admixture). This trend indicates that the features of $1.0 \%$ foreign matter admixture started overlapping with other classes (of admixture).

To assess this overlap, multiple comparisons of means for 70 input features were carried out using Duncan's multiple range test. An overview of comparisons of means revealed that $1.0 \%$ foreign matter admixture had considerable features overlapping with other classes. This overlap led to 

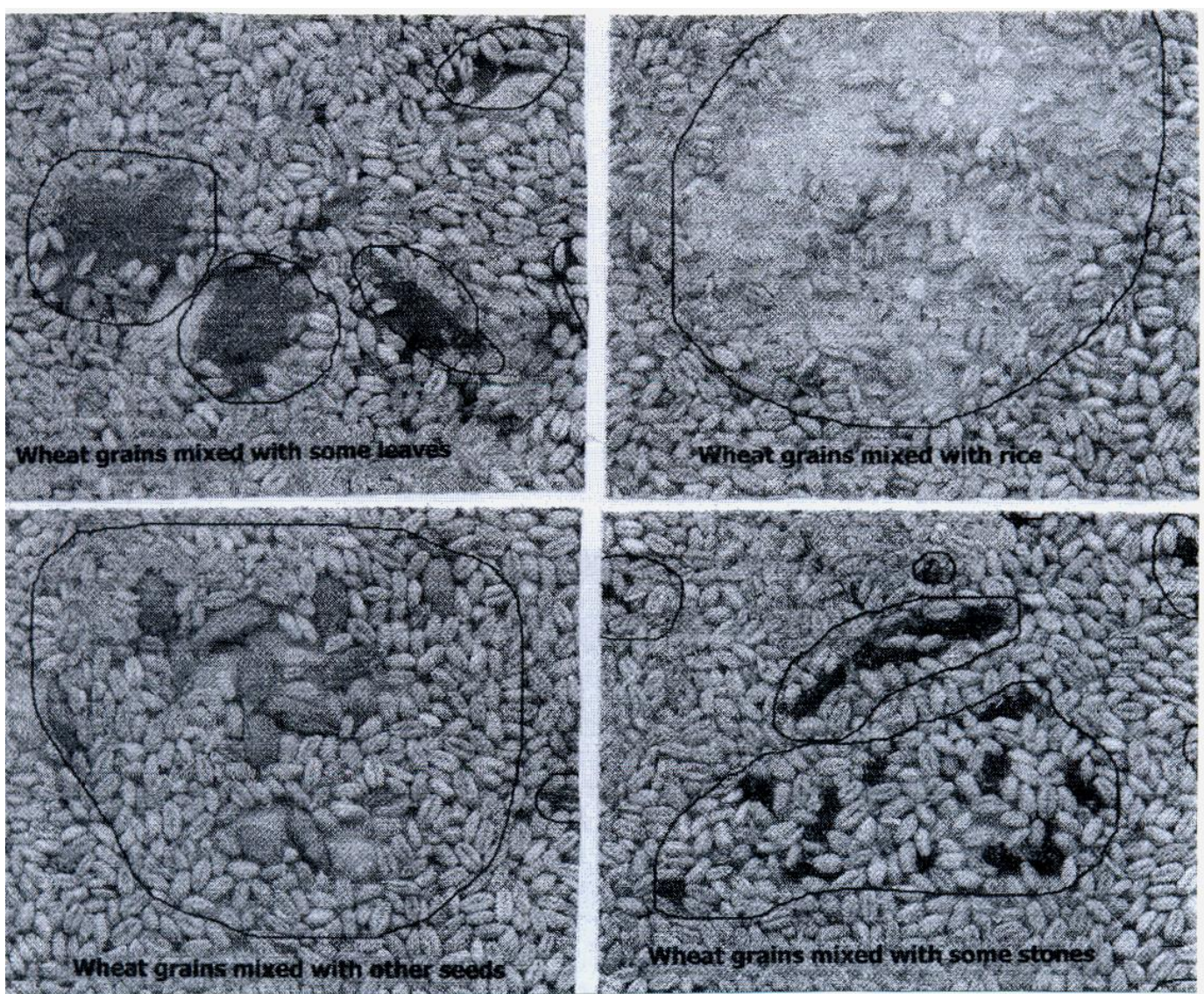

Fig. 1. Wheat samples mixed with foreign matter such as leaves, rice, barley and stones respectively

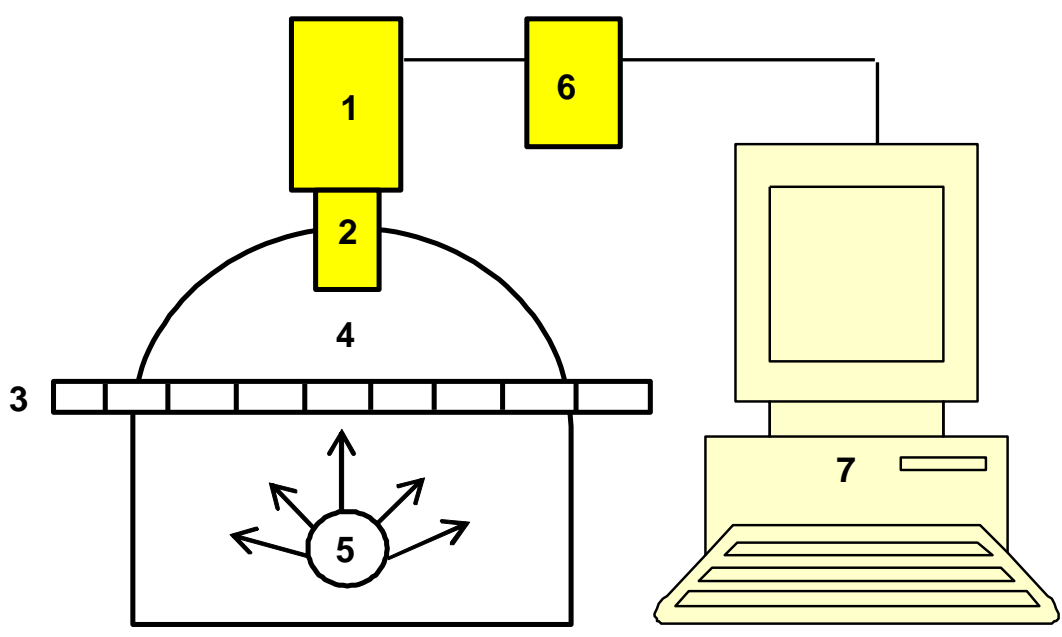

Fig. 2: Diagram of data processing with machine vision system:

1- camera; 2-lens; 3-crate; 4-diffuse dome; 5-backlight; 6-frame grabber and 7- PC(Personal Computer). 


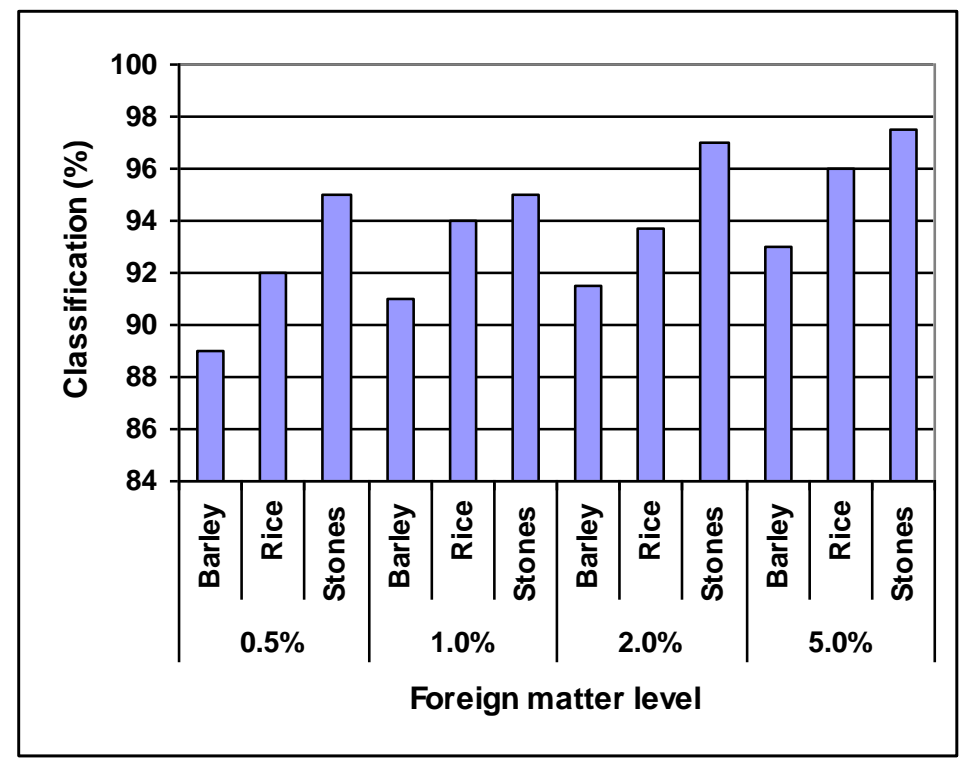

Fig. 3. Classification accuracies of wheat mixed with different percentages of barley, rice and stones using non parametric statistical classifiers

misclassification into other classes as shown in confusion matrix (Table 1). About $11 \%$ of $0.5 \%$ barley admixture was misclassified into next class (1.0\% of barley admixture). On the other hand, $8 \%$ of rice admixture and $5 \%$ of stones admixture were misclassified into next class $(1.0 \%$ of rice and stones admixtures respectively). For $5.0 \%$ foreign matter (admixture with wheat), about $7 \%$ of barley, $4 \%$ of rice and $3 \%$ of stones were misclassified.

That means, $5.0 \%$ foreign matter admixture was better classified than other classes. From these results it is concluded that the algorithm could not classify $1.0 \%$ foreign matter admixtures correctly and requires some modification and testing before it can be recommended for field applications.

\section{Classification using neural network classifiers}

A four layer back propagation neural network (BPNN) was designed with 70 nodes in the first layer, 20 nodes in each of the two hidden layers and 10 nodes in the output layer. Five random training and test data sets were used to calculate the average classification. Fig. (4) depicts about $90 \%, 92 \%$ and $96 \%$ of $0.5 \%$ barley, rice and stones admixtures (with wheat) were accurately classified. On the other hand, 94\%, 95\% and 97\% of $5 \%$ barley, rice and stones admixtures (with wheat) were accurately classified.
There is also a little overlap to a next class as shown in Table (2). About $10 \%$ of $0.5 \%$ barley admixture was misclassified into next class $(1.0 \%$ of barley admixture). On the other hand, $8 \%$ of rice admixture and $4 \%$ of stones admixture were misclassified into next class $(1.0 \%$ of rice and stones admixtures respectively). For $5.0 \%$ foreign matter (admixture with wheat), about $6 \%$ of barley, $5 \%$ of rice and $3 \%$ of stones were misclassified. That means, $5.0 \%$ foreign matter admixture was better classified than other classes. From these results it is concluded that the algorithm could classify $1.0 \%$ foreign matter admixtures correctly compared with statistical classifier (shown in Table 1).

\section{Classification using machine vision}

A machine vision method has recently been developed to detect individual kernel along with individual foreign matter in wheat samples. Image capture (256 x 256 pixels) was using a monochrome CCD camera and PC (personal computer) fitted with frame grabber and the lighting was from a circular fluorescent tube. The detection scheme consists of a hybrid adaptive thresholding algorithm, incorporating median filtering followed by erosion. An example of the process, for a sample of wheat containing some pieces of foreign matter. Detection rates for the contaminants in otherwise clean wheat were $99 \%$ for rice, $98 \%$ for stones and $87 \%$ for barley admixture ( $5 \%$ admixture). 
Table 1. Confusion matrix of statistical classifier showing misclassification. B(for barley), $\mathbf{R}$ (for rice) and $\mathbf{S}$ (for stones)

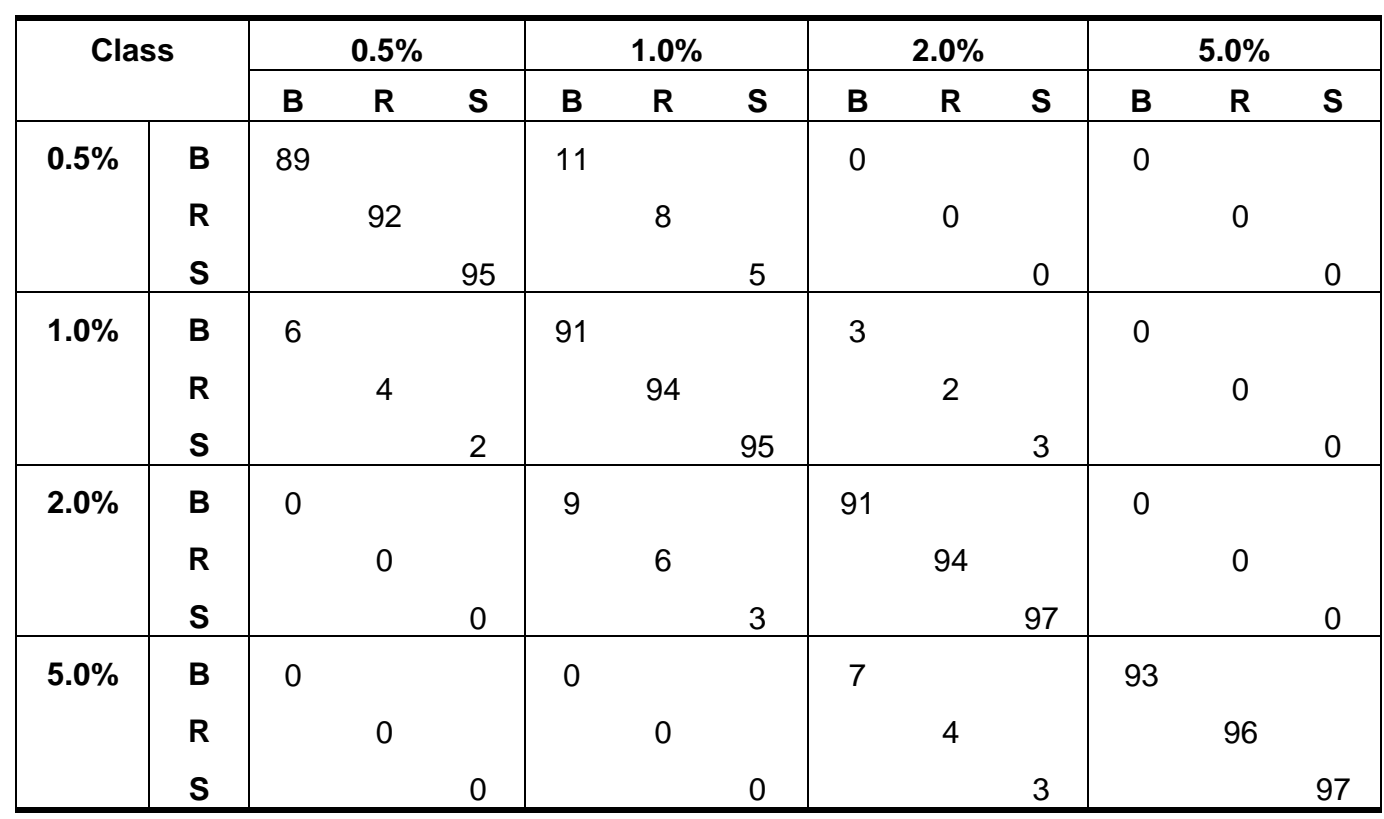

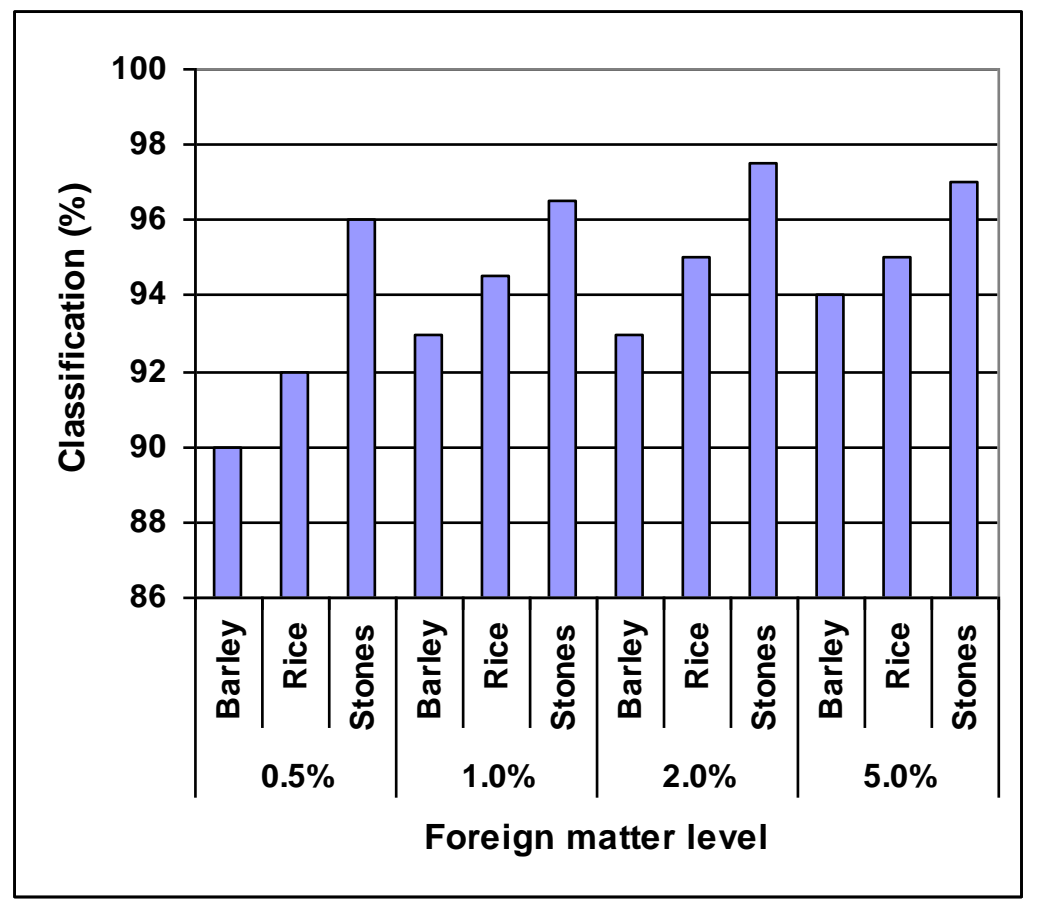

Fig. 4. Classification accuracies of wheat mixed with different percentages of barley, rice and stones using back propagation neural network (BPNN) for full features model. 
Table 2. Confusion matrix of BPNN classifier for full feature model showing misclassification. B (for barley), R (for rice) and S (for stones)

\begin{tabular}{|c|c|c|c|c|c|c|c|c|c|c|c|c|c|}
\hline \multicolumn{2}{|c|}{ Class } & \multicolumn{3}{|c|}{$0.5 \%$} & \multicolumn{3}{|c|}{$1.0 \%$} & \multicolumn{3}{|c|}{$2.0 \%$} & \multicolumn{3}{|c|}{$5.0 \%$} \\
\hline & & B & P & $\mathbf{S}$ & $\mathrm{E}$ & $\mathbf{R}$ & $\mathbf{S}$ & B & $\mathbf{R}$ & $\mathbf{S}$ & B & $\mathbf{R}$ & S \\
\hline $0.5 \%$ & $\mathbf{R}$ & 90 & 92 & 96 & 10 & 8 & 4 & 0 & 0 & ) & 0 & 0 & 0 \\
\hline $1.0 \%$ & B & 0 & 0 & 4 & 93 & 95 & 96 & 7 & 5 & ) & 0 & 0 & 0 \\
\hline $2.0 \%$ & $\mathbf{R}$ & 0 & 0 & 0 & 7 & 5 & 3 & 93 & 95 & 97 & 0 & 0 & 0 \\
\hline $5.0 \%$ & $\mathbf{R}$ & 0 & 0 & 0 & 0 & 0 & 0 & 6 & 4 & 3 & 94 & 96 & 97 \\
\hline
\end{tabular}

Using the same image capture set-up as described above, a second detection scheme was devised for the detection of foreign matter in wheat (such as barley, rice or stones).

This detection scheme was based on a linear feature detector incorporating two orthogonal masks (Chambers et al 1998). An example is given in Fig. (5), for wheat sample containing some foreign matter in wheat. Here, sample presentation was modified slightly and wheat kernels were mostly non-touching to minimize the possibility of foreign matter being obscured by the grain. Here, major effort has been put into algorithm speed up through systematic modification and refinement of the detection schemes and algorithm coding. The detection rate for foreign matter in otherwise clean wheat was $100 \%$ with no false positives.

\section{CONCLUSION}

Classification accuracy using non parametric statistical classifier reduced from $97 \%$ for wheat mixed with stones to $96 \%$ for wheat mixed with rice and $93 \%$ for wheat mixed with barley (at $5.0 \%$ admixture).This trend indicates that the features of $1.0 \%$ foreign matter admixture started overlapping with other classes (of admixture). On the other hand, $94 \%, 95 \%$ and $97 \%$ of $5 \%$ barley, rice and stones admixtures (with wheat) were accurately classified using neural network classifier. Classification accuracy using Back propagation neural network model (BPNN) was higher than the non parametric statistical classifier.

With the level of automation at every level of food production and the rates at which food is being produced, it is becoming increasingly more important to have systems that can automatically detect foreign matter along the way. The color scheme could make a computer vision system very practical for foreign object detection and removal. The application of machine vision for finding foreign matter in wheat samples was studied. Multi-spectral information from histograms of Red, Green and Blue, saturation and lightness images (256 $\times 256$ pixels) was used as an input to multivariate statistical analysis to locate sub-images belonging to foreign matter in wheat samples. The detection scheme consists of a hybrid adaptive thresholding algorithm, incorporating median filtering followed by erosion. Using machine vision system, the detection rate for foreign matter in otherwise clean wheat was $100 \%$ with no false positives. This detection scheme was based on a linear feature detector incorporating two orthogonal masks.

We recommended that, further image processing refinement of the algorithm can be developed onto a production line to test long-term performance for identification of foreign matter in wheat before milling. 


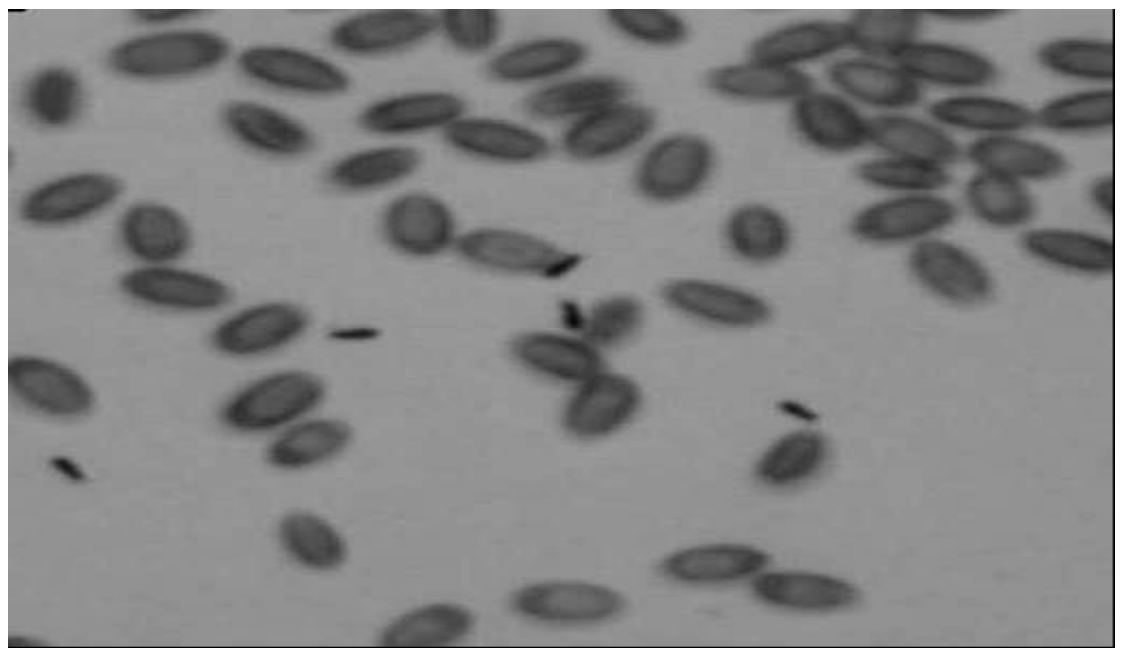

(a)

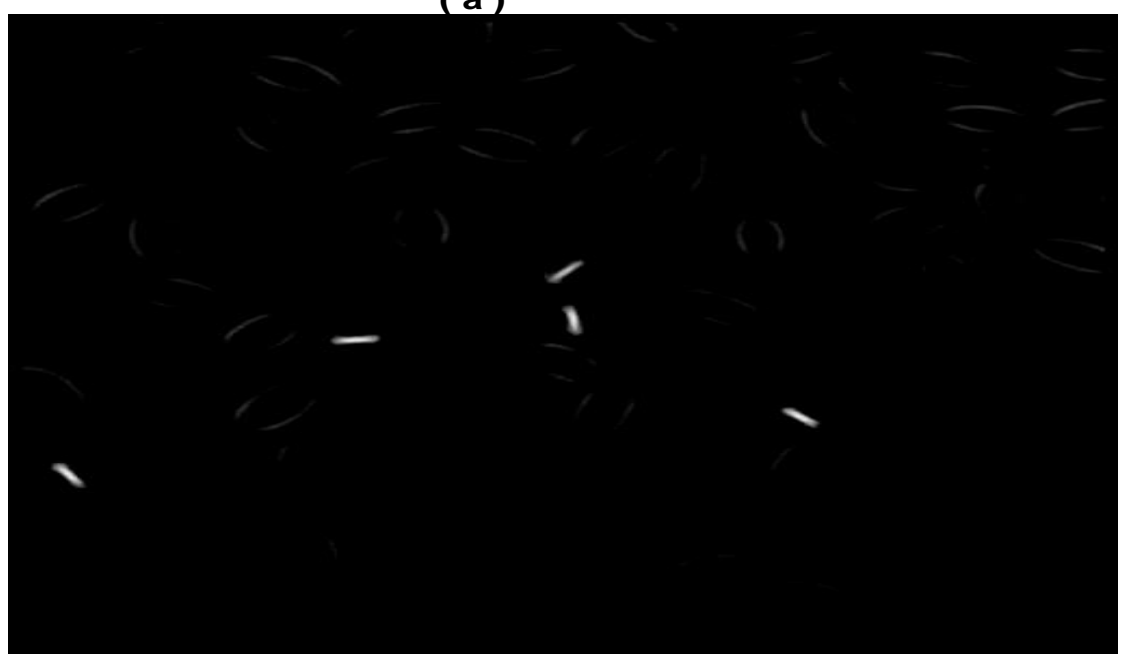

(b)

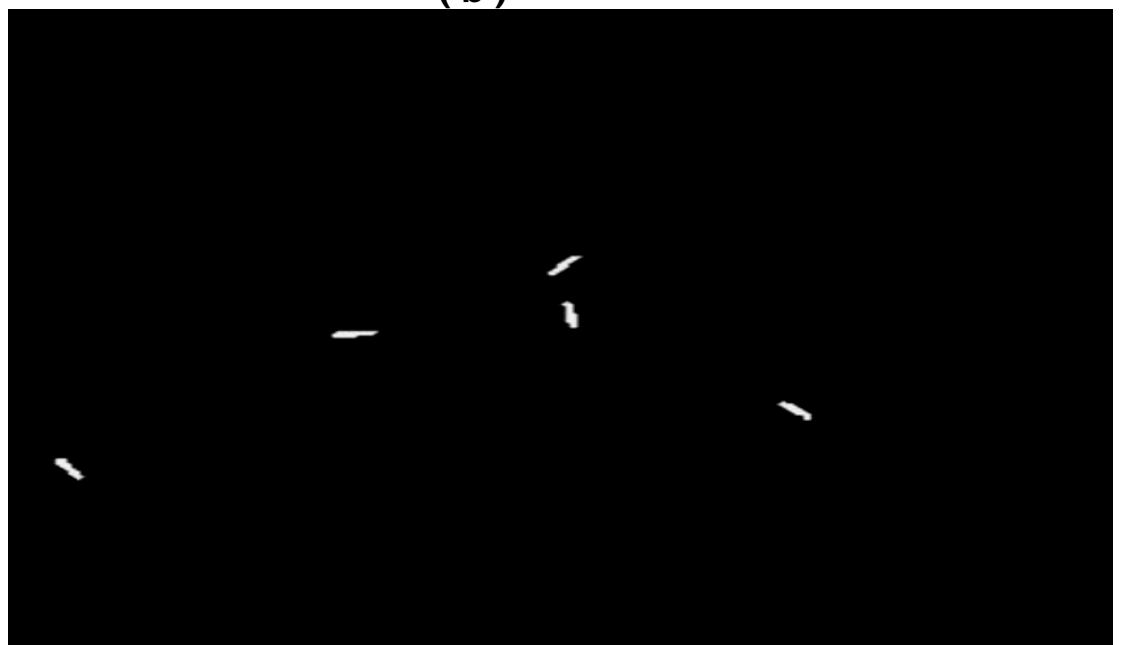

(c)

Fig. 5. Detection of foreign matter in wheat. (a) original image, (b) thresholded image and (c) result of slight erosion of (b).

Arab Univ. J. Agric. Sci., 16(2), 2008 


\section{REFERENCES}

Chambers, J.; C. Ridgway; E.R. Davies; D.R. Mason and M. Bateman (1998). Rapid Automated Detection of Insects and Certain other Contaminants in Cereals. pp. 45-59, HGCA Project, Report No.156, London, UK.

Daley, W.D. and R. Carey (1991). Color Machine Vision for Defect Detection: Algorithm and Techniques. Proc. RIA International Robots \& Vision Automation Conference. pp. 211-217, Tokyo, Japan.

Elbatawi, I.E. (2008). An Acoustic Impact Method to Detect Hollow Heart of Potato Tubers. Journal of Biosystems Engineering, 100(2): 206-213.

Elbatawi, I.E. and M.T. Ebaid (2006). A new Technique for Grape Inspection and Sorting Classification. Arab Universities Journal of Agricultural Sciences, 36: 555-573, Ain Shams University, Cairo, Egypt.

Kenmochi, Y. (1992). A Basic Study of Sensor for Detecting Foreign Material in Food. Sensor Technical, 12(5): 54-60.

Ministry of Agriculture (2003). Wheat Production in Egypt. Technical Report No. 16. Cairo, Egypt. Morita, K.; S. Tanaka; Y. Ogawa and C. Thai (1996a). Detection of Non-Metallic Foreign Materi- als in Food by Soft X-Ray System. American Soc. of Agric. Eng., ASAE paper No. 96-6059, St. Joseph, MI, USA.

Morita, K.; S. Tanaka; Y. Ogawa and C. Thai (1996b). Application of X-Ray CT for Detection of Physical Foreign Materials in Food. American Soc. of Agric. Eng., ASAE paper No. 96-6058, St. Joseph, MI, USA.

Muramatsu, I. (1989). Foreign Material in Food. 32(18): 20-26, Shokuhin Kogyo, Japan

Paliwal, J.; N.S. Visen; D.S. Jayas and N.D.G. White (2003). Cereal Grain and Dockage Identification Using Machine Vision. Biosystems Engineering, 85(1): 51-57.

Petracci, M.; M. Bianchi; M. Betti; M.A. Cremonini; L. Laghi; C. Cavani and G. Placucci (2004). Relationships Between Raw Broiler Breast Meat Color and Low-Resolution NMR Relaxation Properties. Monitoring and Controlling the Plant. American Soc. of Agric. Eng., ASAE paper No. 04-0782, St. Joseph, MI, USA.

SAS (2000). SAS User's Guide Statistics. Ver. 8, $3^{\text {rd }}$ Ed., Vol. 1. Cary, SAS Institute Inc., North Carolina (NC), USA.

Stier, R.F. (2003). The Dirty Dozen: Ways to Reduce the 12 Biggest Foreign Materials Problems. Food Safety Magazine, 64(3): 124-132. 